\title{
Dual Prediction-based Reporting for Object Tracking Sensor Networks
}

\author{
Yingqi Xu Julian Winter Wang-Chien Lee \\ Department of Computer Science and Engineering \\ Pennsylvania State University \\ University Park, PA 16802 \\ E-Mail: $\{$ yixu, jwinter, wlee $\} @$ cse.psu.edu
}

\begin{abstract}
As one of the wireless sensor network killer applications, object tracking sensor networks (OTSNs) disclose many opportunities for energy-aware system design and implementations. In this paper, we investigate prediction-based approaches for performing energy efficient reporting in OTSNs. We propose a dual prediction-based reporting mechanism (called DPR), in which both sensor nodes and the base station predict the future movements of the mobile objects. Transmissions of sensor readings are avoided as long as the predictions are consistent with the real object movements. $D P R$ achieves energy efficiency by intelligently trading off multi-hop/long-range transmissions of sensor readings between sensor nodes and the base station with one-hop/short-range communications of object movement history among neighbor sensor nodes. We explore the impact of several system parameters and moving behavior of tracked objects on DPR performance, and also study two major components of DPR: prediction models and location models through simulations. Our experimental results show that DPR is able to achieve considerable energy savings under various conditions and outperforms existing reporting mechanisms.
\end{abstract}

\section{Introduction}

Recently, the rapid developments of various technologies for sensing, computing and communication have brought a lot of momentum to the research in wireless sensor networks. Even though many energy optimization techniques, e.g., dynamic voltage scheduling [27], energy-aware real time scheduling [10], topology management [3] and intelligent query processing techniques [25], etc. have been studied for sensor networks, they do not fit the object tracking sensor networks (OTSNs) very well. OTSNs imply intricate collaborative operations amongst sensor nodes and produce immense raw streaming data flowing inside the network.
Therefore, many technical and research challenges are faced for incorporating energy awareness into OTSNs.

Object tracking sensor networks have two critical operations: 1) monitoring: sensor nodes are required to detect and track the movement states of mobile objects; 2) reporting: the nodes that sense the objects need to report their discoveries to the applications. These two operations are interleaved during the entire object tracking process. Our focus, in a prior study [23, 24], has been on developing strategies for reducing the energy consumption in monitoring operations. In this paper, we expand our study to the energy management in reporting operations. We propose a dual prediction reporting (DPR) mechanism, which reduces the energy consumption of radio components by minimizing the number of long distance transmissions between sensor nodes and the base station with a reasonable overhead. In DPR, both the base station and sensor nodes make identical predictions about the future movements of mobile objects based on their moving history. By monitoring the mobile objects, the sensor nodes can decide whether or not the predictions reflect the real states of object movements. If the prediction is consistent with monitored states, no update needs to be sent to the base station, which has also correctly predicted the current object movements. Otherwise, the sensor nodes are responsible for correcting the inaccurate predictions performed at the base station.

This paper makes three significant contributions.

- We propose and architect a prediction-based reporting mechanism for OTSNs, namely DPR.

- We employ and compare several different location models in DPR. The granularity and transmission overhead of location models represent a primary tradeoff between the precision (and thus prediction accuracy) and the total energy consumption of DPR.

- We conduct an extensive performance evaluation by simulating DPR with various location 
models and compare them against two existing reporting mechanisms (i.e., naive and PREMON). The results reveal profound insights for designing an energy-aware reporting mechanism in power-constrained OTSNs.

The idea of utilizing predictions to improve system performance in mobile computing environments have appeared in the literature. In cellular environments, paging overhead is reduced by limiting the search space to a set of cells that a mobile user is predicted to possibly enter [1, 20]. Similarly in sensor networks, PREMON reduces the transmissions from sensor nodes by predicting their readings at the base station [4]. For wireless data broadcast, mobile computers are able to predict the arrival time of the requested data frames and thus, selectively retrieve data to reduce power consumption $[6,9,16]$. For Moving Object Database (MOD), Wolfson et. al. propose a framework between MOD and location update stream, which inserts the location changes into MOD only when it does not predict objects' movements correctly [22].

The rest of the paper is organized as follows. Section 2 provides a background for this paper. In Section 3, we propose the dual prediction reporting mechanism and discuss the possible prediction and location models. Section 4 presents the simulation results; and finally, Section 5 lists our conclusion and future work.

\section{Object Tracking Sensor Networks}

Object tracking sensor networks (OTSNs) have widespread use in applications such as security surveillance and wildlife habitat monitoring [18]. OTSNs face severe energy limitations since they usually are driven by scarce energy resources. Therefore, a new networking paradigm for OTSNs, with a focus on the energy efficiency of reporting operations is studied in this paper. In the following, we first provide some background of OTSNs for this paper, and describe a monitoring mechanism underlying the DPR mechanism.

\subsection{Background}

In an OTSN, a number of sensor nodes are deployed over a monitored region with predefined geographical boundaries. The base station acts as the interface between the OTSN and applications by issuing commands and collecting the data of interests. A sensor node has the responsibility for tracking the object intruding its detection area, and reporting the states of the mobile objects with certain reporting frequency, which is adjustable to the network and application requirements. Deciding the states of the tracked objects may need several sensor nodes to work together. This is an important area of research (called sensor fusion) in the sensor applications, but out of scope of this study. Thus, in this paper, we assume that each sensor node is a logical representation (i.e., sensing leaders or cluster heads) of a set of sensor nodes which collaboratively decide the state of a mobile object. Moreover, the sensor nodes are assumed to be static and the base station has a good knowledge about the location of each sensor node during the operating period.

We also assume that the mobile objects are identifiable so that sensor nodes are able to store the mobile objects' movement history, which is used for make predictions for objects' future movement. Some existing techniques are available for identifying the tracked objects, e.g., the objects are electronically tagged or can be identified based on a pre-embedded object code table [7] in the sensor nodes. Also, a low-energy paging channel [12] exists for a sensor node to wake up other sleeping nodes. This channel is deployed for minimizing the energy consumed by the idle state of sensor radio component. Without this channel, the sensor nodes have to be awake all the time. However, this wakeup channel scheme is not directly related to DPR scheme, since the DPR is focused on minimizing the energy consumed by communication operations.

\subsection{Impacting Factors}

In this section, we discuss various factors that have impacts on the energy consumption in OTSN:

- Network workload is related to the number of mobile objects inside the network, which has an impact on the overall energy dissipation of OTSNs due to the amount of monitoring and reporting activities.

- Reporting frequency of sensor nodes is determined by application requirements. Since higher reporting frequency implies more update packets, keeping reporting frequency as low as application allows can conserve energy of OTSNs.

- Data precision is closely relevant to the location models (see below) and the frequency of sampling performed by the sensor nodes. The application can depict the mobile objects' movement in more details by asking for the location information more frequently and/or a location model with higher granularity, which in turn consumes extra energy and requires more computations.

- Location models are used to present the location information of mobile objects. Additional movement states, e.g., speed and direction, can be derived from the location changes. 


\subsection{Monitoring Mechanism}

In a previous study [24], the authors studied several energy-saving schemes for monitoring operations. A prediction-based energy saving scheme (called PES) for monitoring has been shown to achieve high energy efficiency by minimizing the energy dissipation in the micro-controller unit (MCU) and sensor components. However, the energy-saving schemes for monitoring are orthogonal to the reporting mechanism we are addressing in this paper. To focus on the energy optimization for reporting operations, we adopt one of the basic monitoring schemes, called continuous monitoring scheme (CM) which was also discussed in [24]. This scheme activates a sensor node only when an object enters its detection area and keeps the node awake for monitoring until the object leaves its cell. The advantage of CM is that it involves only one sensor node for monitoring each mobile object, while other sensor nodes can switch to sleep mode and save energy. However, to ensure no missing reports, the active sensor has to stay awake all the time as long as the object stay in its detection area, even if the application requires only a very low reporting frequency.

\section{Dual Prediction based Reporting}

The characteristics of energy dissipation on a wireless sensor node for monitoring and for reporting are very different $[5,14]$. In the monitoring stage, the MCU and sensor components dominate the energy consumption and are the main energy optimization targets, which represents the design philosophy of [24]. For reporting, while the MCU still participates the communications in terms of decoding and encoding the packets, the main part of energy dissipation has shifted to the radio components. To transmit sensor discoveries back to the base station, the network involves a large number of radio operations, including listening to the channels, receiving data and transmitting data.

Based on an observation that the overall direction of an object's movement usually does not change dramatically during its trip and thus can be predicted from past moving patterns, we propose a dual prediction based reporting (DPR) mechanism to conserve energy at the reporting stage of an OTSN.

\subsection{Reporting Mechanism}

The DPR mechanism has two main components: location models which regulate the granularity of the location information that the system desires; and prediction models that analyze the moving history of tracked objects and estimate their future movement states. In the DPR mechanism, a prediction model is deployed at both sensor nodes and the base station. By using the same his- torical data, the sensor nodes and the base station consistently make the same prediction of any mobile object's future movements. This way, the sensor nodes are able to avoid transmitting its sense data to the base station, as long as their predictions about the object's movements match their readings. Meanwhile, the base station always assumes its prediction reflects the real movements of mobile objects, unless it receives the corrections (called update packets) from the sensor nodes.

To make predictions, the moving history of all objects is needed. The base station can obtain these historical data either from its own correct predictions, or the update packets from the sensor nodes (when its predictions were incorrect). For the sensor nodes, however, they do not have a global view of the entire network as the base station does. Therefore, historical packets ${ }^{1}$ have to be passed among the sensor nodes who are involved in tracking objects. Compared with the long distance/multi-hop transmissions from sensor nodes to the base station, exchanging historical packets locally consumes much less energy. Therefore, the overhead of exchanging historical packets represents a good tradeoff for the gain of DPR mechanism, which is verified by our experimental results (Section 4).

The basic idea of DPR is that a sensor node which has a mobile object in its territory first predicts the movement of the mobile object for the next reporting period. Meanwhile, the base station makes the same prediction based on the same objects' movement history. The predictions at the base station, stored locally, are never transmitted to the sensor nodes and thus incur no communication cost. If the observed object's movement matches the sensor node's prediction, no transmission is needed, since the base station has the same predictions as the sensor node. Otherwise, the sensor node has to correct the base station by sending the real objects' movement, so that the base station can update its cached object movement history. In this way, the history stored at the base station and sensor nodes are consistent, and their predictions for the objects' movement are identical all the time. The transmissions from the sensor nodes to the base station are avoided as long as the predictions are correct. On the other hand, to allow the sensor nodes which never saw the object to make predictions, the objects' movement history has to be passed between sensor nodes as the object moves. Even though exchanging movement history consumes extra communication overhead, these one-hop transmissions among neighbor

1 The moving history of mobile objects can be compressed by the prediction models (discussed in Section 3.2). Therefore, obtaining these historical data does not incur excessive storage and communication overheads, even when multiple objects have to be tracked at the same time. 
sensor nodes are minor compared with the multi-hop transmissions between the base station and sensor nodes which DPR saves.

The DPR mechanism raises several research issues. For example, due to the fixed cost of historical data exchanges, the overall performance of DPR relies on the accuracy of the prediction mechanism. How can we improve the prediction accuracy? Also, what kinds of historical data could be the input for a prediction model? Intuitively, finer granular historical information allows more accurate estimations that a prediction model may make, although more presentation and communication overheads are incurred. This observation points out a tradeoff between the granularity of the historical data and the accuracy of the prediction model. The remaining of this section will address these questions in details.

\subsection{Prediction Models}

Prediction models refer to the prediction functions that incorporate heuristics and strategies for predicting object movements. If the sensor nodes and the base station can accurately predict all future moves of the objects, there actually is no need for any communication. Thus, the strategies that explore the moving history of mobile objects are vital to a prediction-based scheme.

In our prior work [24], we proposed and studied three prediction models: namely instant, average and exponential average. Basically, the instant model assumes that the object continues to move in the same direction and velocity as the last observed state. The average model predicts an object's future moving states by averaging the historical data. The exponential average model lends more weight to recent history which is more likely to reflect the future behavior. Those prediction models can also be employed for dual prediction based reporting mechanism. Moreover, the prediction models can compress the entire moving history of mobile objects and efficiently reduce the overhead for storing and exchanging historical packets amongst the sensor nodes. Due to the space constraint, we do not provide details here. Interested readers are referred to [24].

\subsection{Location Models}

Prediction models are concerned with how to process the historical data, and use them to predict the next movements of mobile objects. In this section, we discuss the location models which decide the precision of these historical data, thus indirectly affect the accuracy of the prediction models. Meanwhile, the application can choose the appropriate location model for mobile objects' location reported by the sensor nodes.

In a two-dimensional space, given the locations of a moving object at time $t_{i}$ and $t_{j},\left(x_{i}, y_{i}\right)$ and $\left(x_{j}, y_{j}\right)$, respectively, the speed $v_{i j}=\frac{\sqrt{\left(x_{i}-x_{j}\right)^{2}+\left(y_{i}-y_{j}\right)^{2}}}{\left|t_{i}-t_{j}\right|}$ and direction $d_{i j}=\tan ^{-1} \frac{x_{i}-x_{j}}{y_{i}-y_{j}}$ can be computed. From the above equations, we observe that two factors control the precision of the historical data. One is the unit of time (i.e., $\left|t_{i}-t_{j}\right|$ ). A longer time unit hides the minor changes in object movements, since all the variations in the speed and directions between $t_{i}$ and $t_{j}$ are averaged. The second factor is the location model (i.e., $x_{i}$ and $\left.y_{i}\right)$. The location information with higher granularity provides more information about the object's moving history to the prediction models, thus is more likely to achieve higher prediction accuracy. In general, location models can be categorized into two different models: geometric and symbolic [8]. A geometric model provides $n$-dimensional coordinates, which is fine-grained, but incurs considerable cost and complexity. A symbolic model denotes an area as the location of the object. In this paper, we discuss several possible location models (illustrated in Figure 1), and study their effect on the overall performance of DPR.

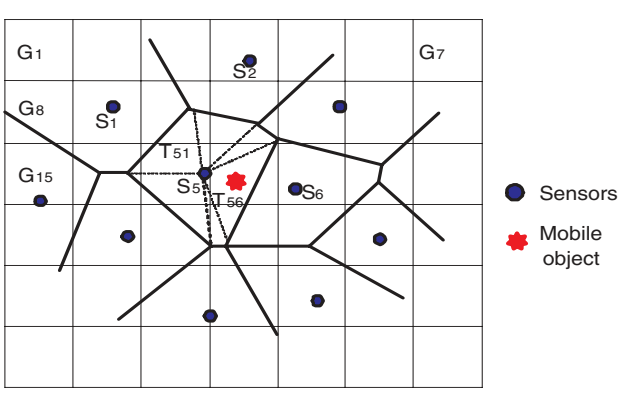

Figure 1. Location Models for DPR

- Sensor cell is the most coarse symbolic location model among the models we discuss. As Figure 1 shows, the Sensor_ID (e.g., $S_{5}$ ) represents the location of the mobile object.

- Triangle is formed by connecting endpoints of the boundary between two adjacent sensor nodes to the center of the sensor cell. For example, $T_{56}$ in Figure 1, the triangle in $S_{5}$ and adjacent to $S_{6}$ represents the location of the mobile object. The advantage of this model is that a sensor node can easily identify the triangle where an object is located in (i.e., involves less sensor fusion activity) and pinpoint the object's location with a smaller symbolic region.

- Grid is another symbolic location model the OTSN can use. In Figure 1, $G_{18}$ indicates the ID of the grid where the object is detected. The total number of grids decides the granularity of the location model. When the number of the grids increases, the 
resulting grid location model approaches the geometric location model (discussed below). However, with a reasonable grid size, grid models can efficiently reduce the representation overhead compared with the coordinates model, but more prediction errors due to the less accurate location information are expected.

- Coordinate belongs to the geometric location model, which holds the most accurate location information. Therefore, the estimation about object movements from this location model is the most accurate one, while the overhead for passing coordinates, which are in the update packets and historical packets increases.

Among the location models we discussed above, Grids are the most flexible. By tuning the grid size, its granularity varies between the coordinates and sensor cells location model. The granularity of the sensor and triangle location models can be improved by increasing the density of sensor nodes. We expect that a location model with higher granularity produces more accurate prediction results, but meanwhile, incurs more transmission overhead in terms of update packets and historical packets.

\section{Performance Evaluation}

In this section, we evaluate the performance of DPR mechanism by simulations. We first describe the model and settings for our simulation. Then, we evaluate how system parameters and moving behavior of monitored objects affect the performance of DPR. Finally, we examine the impact of location models.

\subsection{Simulation Settings}

The DPR mechanism is implemented with all the location models we discussed in Section $3 \cdot 3^{2}$. To simplify our discussion, we assume that the applications require only the Sensor_ID for reporting of object movements. Thus, we identify four possible implementations as SS, ST, SG, and SC, where the first letter represents the location model used in reporting (i,e., Sensor_ID) and the second letter denotes the location model used in prediction (i.e., Sensor, Triangle, Grid and Coordinates). For SG, we assume the monitored area is divided into $100 \times 100$ grids by default, which will be shown to be the best choice for SG in Section 4.5.

Two metrics are used to evaluate the performance of DPR: 1) Total energy consumption measures the total

2 We did simulate the DPR with three prediction models. The results show that the location models have a much higher impact on the performance than the prediction models. For the sake of presentation, we only show the results with INSTANT model and results for other prediction models are available upon request. dissipated energy for reporting operations. Since the focus of this paper is on the reporting mechanism, we only consider the energy cost of radio components; the energy consumed by the MCU and sensor components is neglected. Also the cost of low-power paging channel is negligible (as shown by $[12,26]$ ) and thus not counted; 2) Prediction accuracy measures the ratio of the correct predictions to the total number of predictions made. This metric reflects the energy savings and the overhead of DPR mechanism.

We implemented OTSNs with CSIM [15], a processoriented, discrete-event simulator. We assume the sensor nodes are able to adjust their radio range based on the distribution of sensor nodes. In the experiments, Voronoi Diagram [17] is used to determine the sensing and radio ranges of the sensor nodes. We use the shortest path multi-hop routing algorithm for the communications between the base station and sensor nodes and adopt the energy consumption for WINS sensor nodes $[14,19]$ as the basis for our simulation.

The moving behavior of the tracked objects is modelled by Gauss-Markov mobility model [11]. In this mobility model, the tuning parameter $\alpha$ controls the overall moving trend of mobile object, and also indicates the weight of moving history counted for the future movements. Other parameters, e.g., moving duration, the period of time that objects keep constant moving states, and moving speed also contribute to the randomness of the moving behavior.

In addition to DPR, we also implemented the naive scheme and the PREMON scheme for comparison. In the naive scheme, sensor nodes detect the mobile objects inside their detection regions and report the readings regarding to mobile objects to the base station based on the reporting frequency specified by applications. The nodes which do no have mobile object in their detection area do not report back to the base station. The PREMON scheme [4], also a prediction-based reporting mechanism, assumes that sensors in close proximity are likely to have correlated reading and the base station is able to predict the sensor readings given certain historical and background knowledge. Therefore, the base station makes predictions about the sensor readings and transmits every prediction to the corresponding sensor node. If the predictions received from the base station are correct, the sensor nodes do not need to report their readings, otherwise, they have to update the correct readings to the base station. PREMON prevents a sensor node from unnecessarily transmitting all the readings that can be successfully predicted by the base station, thereby saving energy. In PREMON, sensor nodes do not make predictions, but only monitor the state of mobile object. Thus, no exchange of historical data is nec- 


\begin{tabular}{|l|l||l|l|}
\hline Parameters & Default Settings & Parameters & Default Settings \\
\hline \hline Terrain & $120 \mathrm{~m} \times 120 \mathrm{~m}$ & Number of Nodes & 95 \\
\hline Number of Grids & $100 \times 100$ & Simulation duration & $120 \mathrm{~s}$ \\
\hline Number of trials & 25 & Reporting period & $1 \mathrm{~s}$ \\
\hline Transmission energy & $720 \mathrm{~mW}$ & Receiving energy & $369 \mathrm{~mW}$ \\
\hline Objects - moving speed & $5 \mathrm{~m} / \mathrm{s}$ & Objects - moving duration & $0.6 \mathrm{~s}$ \\
\hline Objects - $\alpha$ & 0.95 & Prediction model & Instant \\
\hline Naive-update packets & 88 bits & Naive - wakeup packets & 56 bits \\
\hline PREMON - update packets & 112 bits & PREMON - wakeup packets & 56 bits \\
\hline PREMON - prediction packets & 88 bits & & \\
\hline DPR-SS - update packets & 88 bits & DPR-SS - historical packets & 88 bits \\
\hline DPR-ST - update packets & 104 bits & DPR-ST - historical packets & 88 bits \\
\hline DPR-SG - update packets & 104 bits & DPR-SG - historical packets & 88 bits \\
\hline DPR-SC - update packets & 112 bits & DPR-SC - historical packets & 96 bits \\
\hline
\end{tabular}

Table 1. System Parameters

essary.

We assume there is no congestion or communication collisions in the network. Exploring the behavior of DPR under congestion and collisions is the subject of our future research. Table 1 summarizes the system parameter settings in our simulations.

\subsection{Network Workload}

Our first experiment compares DPR with different location models to naive and PREMON by varying the network workload, which is captured by the number of mobile objects. Figure 2(a) shows PREMON costs almost as much energy as the naive scheme, if not more. It is because even though PREMON reduces the number of updates from sensor nodes to the base station, it incurs more transmissions from the base station to the corresponding sensor nodes. As a result, without high prediction accuracy PREMON cannot outperform our naive system. This remark is further demonstrated in the following experiments.

DPR with different location models are noticeably more energy efficient than the naive and PREMON schemes. DPR with ST and SS produce an approximately $30 \%$ energy consumption reduction over the naive scheme, and SG consumes even less than half of the energy costed by the naive scheme. DPR achieves significant energy savings by reducing the number of long distance transmissions with relatively low overhead of additional historical packets transmitted among neighbors nodes. In addition, SG and SC benefit considerably from finer location models. Their high prediction accuracy efficiently reduce the number of update packets and energy consumption, regardless of the higher overhead in the update and historical packets.

In Figure 2(b), while ST is 15\% more accurate in predicting the object movements than SS, ST merely outperforms SS with less than $0.1 \%$ energy savings. This shows a tradeoff between the granularity of the location models and the prediction accuracy. The energy savings

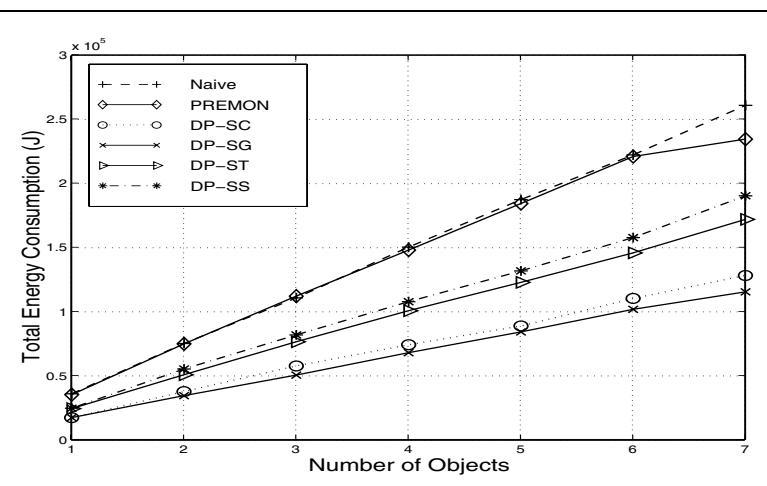

(a) Total Energy Consumption

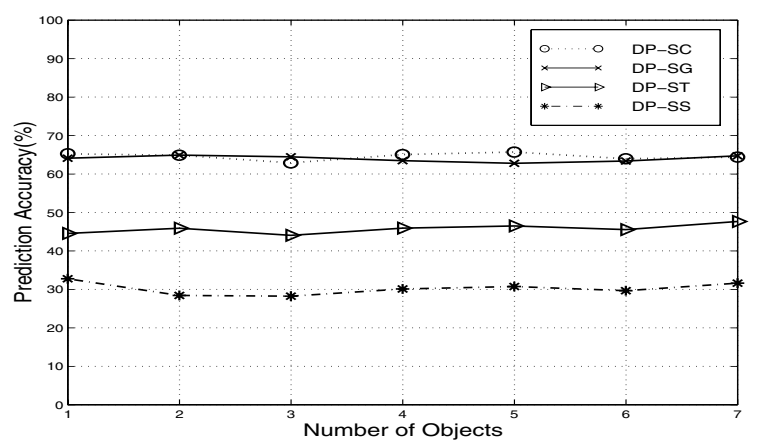

(b) Prediction Accuracy

Figure 2. Impact of the network workload

achieved from the higher predication accuracy in ST do not dramatically overcome the transmission overheads that comes with the finer location models.

Both Figure 2(a) and Figure 2(b) show that the performance of DPR is not sensitive to the number of mobile objects, which implies that DPR scales well with the network load under the assumption that the network is collision free, while studying the performance of DPR under communication collisions will be our next step. However, as one could expect, with the number of ob- 
jects inside the network increases, communication collisions may possibly happen. These collisions may reduce sensor nodes' prediction accuracy and increase the number of updates, which sensor nodes have to transmit to the base station, thus consuming more energy. Therefore, it is indeed interesting to see how well PDR fares under this situation.

For the rest of experiments, we set the number of objects in the network to 1 .

\subsection{Impact of Moving Behavior}

To study the impact of object moving behavior on DPR, we focus on two controlling parameters: the moving duration and the moving speed. The moving duration controls the frequency that objects change their moving states. A longer moving duration implies that an object keeps constant speed and direction for a longer time, thus facilitating the predictions. The moving speed indicates the movement range within the unit of time. Intuitively, the movements of the objects with high speed is hard to capture by the prediction models.

4.3.1. Impact of Moving Duration. To simulate various moving behaviors, we change the movement states (i.e., moving speed and directions) of mobile objects from up to 20 times per second (i.e., moving duration $=50 \mathrm{msec}$ ) to once every 50 seconds (i.e., moving duration $=50000 \mathrm{msec}$ ). In the worst case, DPR is still able to predict at least $25 \%$ of object movements (shown in Figure 3(b)) and achieves reasonable energy savings. In general, with increasing moving duration, DPR with all location models moderately reduce the energy consumption because an object's stable movement helps to improve the prediction accuracy. However, the following insights are revealed from in-depth analysis of the experimental results:

- Moving duration affects the number of historical packets. As moving duration decreases, the movement of mobile objects tends to follow Brownian motion [2], in which the object returns to the origin with high probability $[13,21]$. Thus, the object with smaller moving duration is more likely to revisit the same sensor node multiple times, thus causing more historical packet transmissions.

- Moving duration has an impact on prediction accuracy as well. Figure 3(b) shows that with the frequent transition of movement states (e.g., moving duration is $50 \mathrm{msec}$ ), the prediction accuracy of SC is merely $34 \%$. This ratio radically increases, even up to $100 \%$, as the moving duration gets larger. Correspondingly, the energy consumption for the SC is reduced up to $90 \%$.

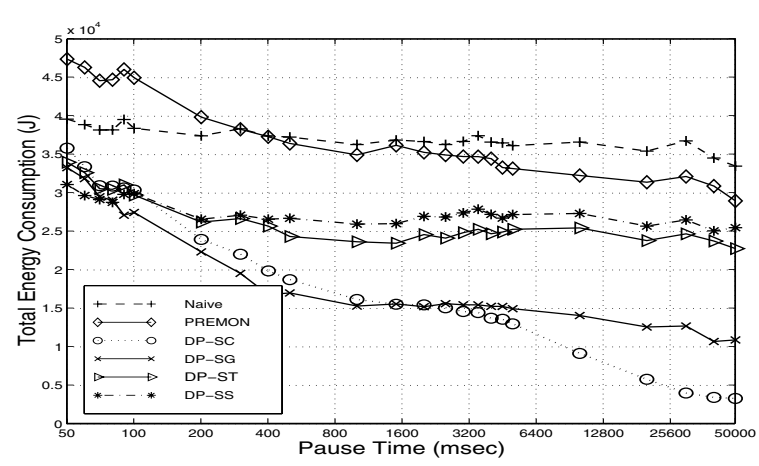

(a) Total Energy Consumption

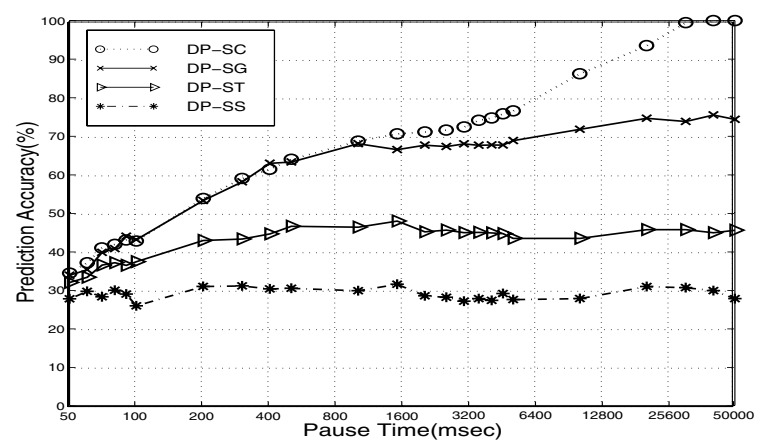

(b) Prediction Accuracy

Figure 3. Impact of Moving Duration (note: $x$-axis is in logarithmical scale.)

- The fine-grained location models are more sensitive to the changes of moving duration than the coarse ones. As we observed from Figure 3(b), SG and SC have more visible improvements of prediction accuracy and energy consumption than ST and SS.

4.3.2. Impact of Moving Speed. Figure 4 plots DPR performance as a function of moving speed of mobile objects. Basically, the energy consumption of all schemes increase as the mobile object move faster. There are two reasons behind this. First, the faster an object moves, the more sensor nodes the object may pass through during the simulation period, which results in more historical packets and energy consumption. Second, a higher moving speed expands the objects possible moving range, thus making predictions of objects' future movements less accurate and triggers more update packets. However, DPR-SS in Figure 4(a) shows approximately constant energy consumption with increasing prediction accuracy. The explanation for this exists within the location models. Because of the low granularity of SS, the prediction is made based on the coordinates of the sensor node where the object was 


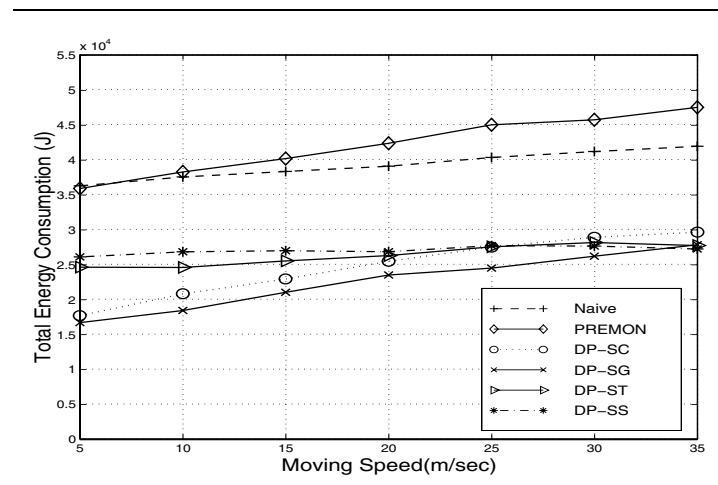

(a) Total Energy Consumption

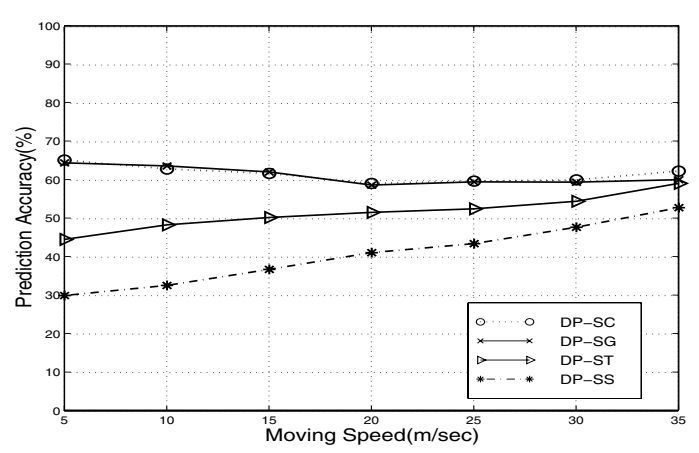

(b) Prediction Accuracy

Figure 4. Impact of Moving Speed

detected, instead of the actual coordinates of the mobile object. Thus, there is a deviation between the actual location of the object and the sensor coordinates used to estimate the location of mobile object for the next report. The effect of this divergence declines when the moving distance between two predictions increases. Moreover, if the object does not move out of the detection area of a sensor node after one prediction period, SS does not treat it as a location change, although the object did change its coordinates in SC model. A mobile object with slower moving speed is more likely to stay in the same sensor cell. In this case, SS is not able to catch the transition of movement states for this object, thus losing more historical information about the object. Increasing the moving speed can help the location models with low granularity to overcome these two side effects, which are termed as granularity effect since they are caused by the granularity of the location models.

Considering the granularity effect, higher object speed assists the location models with low granularity such as ST and SS to achieve higher prediction accuracy. On the other hand, Figure 4 shows that for DPR-SG and DPR-SC, the effect brought by the high moving speed to the predictions overwhelms the granularity effect.

\subsection{Impact of Reporting Period}

The reporting period is one of the most important application requirements to OTSNs, because it not only decides how often the sensor nodes report to the base station in naive scheme, but also represents the prediction period that a prediction-based scheme has to make a prediction for. Lengthening the reporting period impedes prediction-based schemes due to the longer prediction period, but at the same time eases the naive and prediction-based schemes by requesting less update packets and less predictions, respectively. Therefore a longer reporting period is adverse to the predictionbased schemes. This claim is verified by Figure 5, where the energy efficiency of all DPR mechanisms falls off by up to $55 \%$. When the reporting period is over $5000 \mathrm{msec}$, the energy conserved by DPR from the naive scheme is less than 10\%. Figure 5(b) shows SS achieves a higher prediction accuracy as the reporting period prolongs, which is opposite to the other three location models due to the granularity effect. With the extended reporting period, the objects are capable of moving farther during one prediction period, which effectively reduces the impact of low granularity in SS.

\subsection{Impact of Location Models}

In this section, we examine the location model with all levels of granularity by varying the number of grids in SG from $2 \times 2$ to $100 \times 100$. We expect that the energy consumption could be optimized by dividing the monitored area into more grids.

Figure 6(a) shows that the total energy consumption evolves as we expected. DPR-SG outperforms naive, PREMON, DPR-SS, DPR-ST and DPR-SC gradually as the number of grids increases, since the prediction models get more accurate information about objects movement history. However, observing both Figure 6(a) and Figure 6(b), we found that they are not synchronized. For example, when the number of grids equals to 100 , DPR-SG estimates the objects movement as accurate as DPR-SS, but the latter one is more energy cost-effective. It is because that with the same prediction accuracy, the overhead of location models controls the energy consumption. SG becomes most energy efficient when the total number of grids reaches 3600 , because it incurs less communication overhead comparing with DPR-SC with same predication accuracy. However SC represents the finest location model and its prediction accuracy is the upper bound for all location models. Therefore SG can only accomplish limited energy conservation over DPRSC mechanism. 


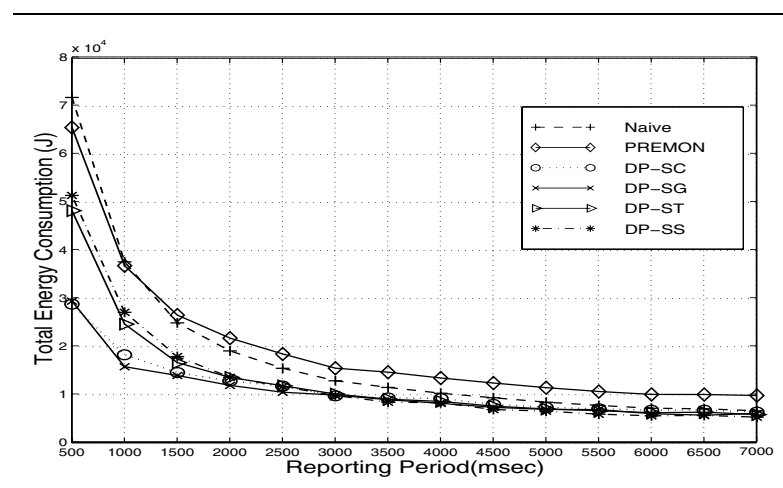

(a) Total Energy Consumption

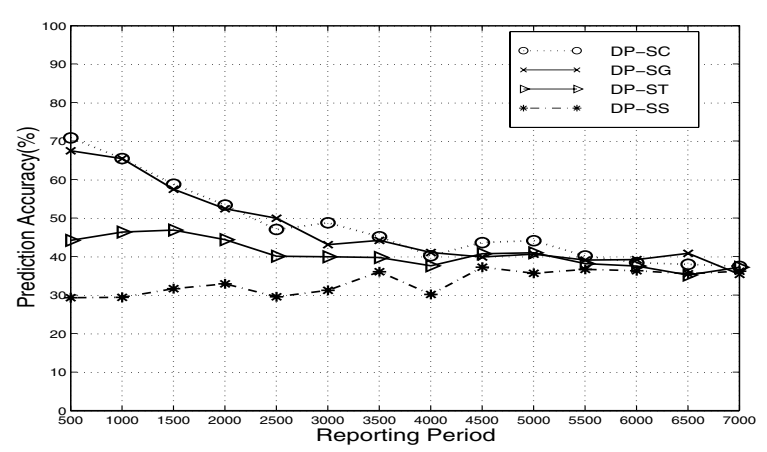

(b) Prediction Accuracy

Figure 5. Impact of Reporting Period

\section{Conclusion and Future Work}

Object tracking sensor networks have two primary operations: monitoring and reporting. This paper addresses the energy conservation issues in the reporting operations. We proposed the Dual Prediction Reporting (DPR) mechanism, in which the sensor nodes make intelligent decisions about whether or not to send updates of objects movement states to the base station and thus save energy. DPR consists of two major components, i.e., location model and prediction model. The choice of a location model determines the granularity of the movement states of mobile objects. A prediction model, on the other hand, decides how to estimate the objects' future movement from their movement history. The performance of prediction based scheme depends on these two components since they govern the precision of historical movement data and the way to process these data.

Extensive simulations have been conducted to evaluate the performance of the DPR and to reveal more insights about choices of location models. The experimental results show several lessons. First, DPR is able to minimize the energy usage of OTSNs efficiently under various condition. Second, the energy savings achieved by DPR are stable under the ranges of mobile object dynamics considered in this paper, even the total energy

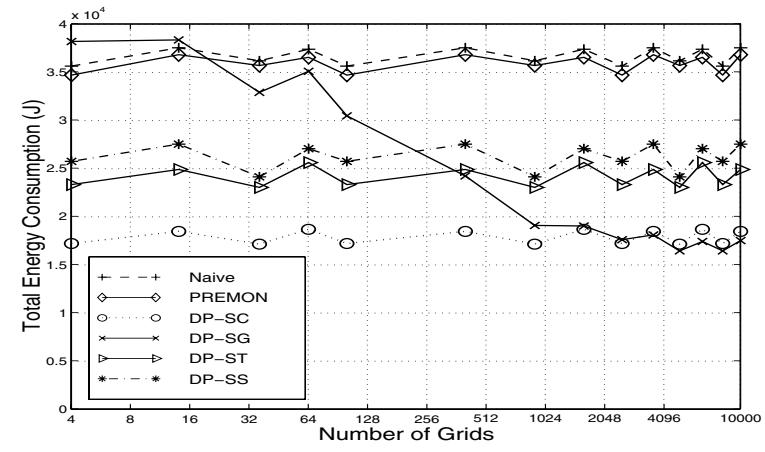

(a) Total Energy Consumption

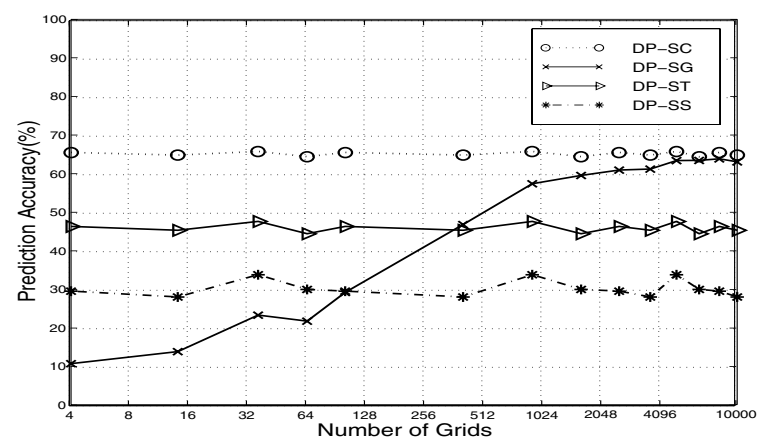

(b) Prediction Accuracy

Figure 6. Impact of Location Models (note: $x$-axis is in logarithmical scale.)

consumption varies. Third, the dynamics of the mobile objects have less impact on the low granular location models than the high granular one due to the granularity effect. Lastly, the longer reporting period is adverse to the prediction-based schemes with high granular location models, but improves the prediction accuracy for the location models with low gutturality by eliminating the granularity effect. The simulations expose the impact of the location models, which facilitates the selection of appropriate location models to meet the application requirements and to address the energy conservation issues.

As for the next steps, we plan to conduct a more extensive evaluation that further studies the impact of sensor detection errors and network communication collisions on the performance of DPR protocol. We are also integrating the DPR mechanism with the PES monitoring mechanism into a complete design of an object tracking sensor network. In addition, we are looking into other network and data management issues in OTSNs, such as topology management, query dissemination and data aggregation. 


\section{Acknowledgements}

The authors would like to thank the anonymous reviewers for their helpful comments, which improved the presentation of this paper. Special thanks go to Prof. Erdal Cayirci at Istanbul Technical University and Dr. Gail Mitchell at BBN, who provided many constructive suggestions on the revision of this paper.

\section{References}

[1] A. Bhattacharya and S. K. Das. Lezi-update: An information-theoretic framework for personal mobility tracking in PCS networks. ACM/Kluwer Journal on Wireless Networks, 8(2-3):121-135, March-May 2002.

[2] T. Camp, J. Boleng, and V. Davies. A survey of mobility models for ad hoc network research. Wireless Communications and Mobile Computing: Special issue on Mobile Ad Hoc Networking: Research, Trends and Applications, 2(5):483-502, 2002.

[3] B. Deb, S. Bhatnagar, and B. Nath. A topology discovery algorithm for sensor networks with applications to network management. In IEEE CAS workshop on Wireless Communication and Networking, Pasadena, CA, September 2002.

[4] S. Goel and T. Imielinski. Prediction-based monitoring in sensor networks: taking lessons from MPEG. ACM Computer Communication Review, 31(5), October 2001.

[5] J. Hill, R. Szewczyk, A. Woo, S. Hollar, D. Culler, and K. Pister. System architecture directions for network sensors. Proceedings of the international conference on Architectural Support for Programming Languages and Operating Systems, pages 93-104, 2000.

[6] T. Imielinski, S. Viswanathan, and B. R. Badrinath. Data on the air - organization and access. IEEE Transactions of Data and Knowledge Engineering, 9(3):353372, 1997.

[7] C. Intanagonwiwat, R. Govindan, and D. Estrin. Directed diffusion: a scalable and robust communication paradigm for sensor networks. In International Conference on Mobile Computing and Networking, pages 56-67, Boston, Massaachusetts, 2000.

[8] D. Lee, W.-C. Lee, J. Xu, and B. Zheng. Data management in location-dependent information services. IEEE Pervasive Computing, 1(3):65-72, 2002.

[9] W.-C. Lee and D. Lee. Using signature techniques for information filtering in wireless and mobile environments. Distributed and Parallel Databases Jounal: Special Issue on Database and Mobile Computing, 4(3):205-227.

[10] H. Li, P. Shenoy, and K. Ramamritham. Scheduling communication in real-time sensor applications. In Proc. IEEE Real-Time and Embedded Technology and Applications Symposium, Toronto, Canada, May 2004.

[11] B. Liang and Z. J. Haas. Predictive distance-based mobility management for PCS networks. In The 22nd Annual Joint Conference of the IEEE Computer and Communications Societies(INFOCOM), pages 1377-1384, 1999.
[12] M. J. Miller and N. H. Vaidya. Minimizing energy consumption in sensor networks using a wakeup radio. In Proc. IEEE Wireless Communication and Networking Conference, Atlanta, GA, 2004.

[13] S.C. Port and C.J. Stone. Brownian motion and classical potential theory. Academic Press, New York, 1978.

[14] V. Raghunathan, C. Schurgers, S. Park, and M. B. Srivastava. Energy aware wireless microsensor networks. IEEE Signal Processing Magazine, 19(2):40-50, 2002.

[15] H. Schwetman. CSIM user's guide (version 18). Mesquite Software, Inc., http://www.mesquite.com.

[16] N. Shivakumar and S. Venkatasubramanian. Efficient indexing for broadcast based wireless systems. ACM/Baltzer Mobile Networks and Applications (MONET), 1(4):433 - 446, December 1996.

[17] I. Stojmenovic, A. P. Ruhil, and D. K. Lobiyal. Voronoi diagram and convex hull based geocasting and routing in wireless networks. In The IEEE Symposium on Computers and Communications, Antalya, Turkey, July 2003.

[18] R. Szewczyk, E. Osterweil, J. Polastre, M. Hamilton, A. Mainwaring, and D. Estrin. Application driven systems research: Habitat monitoring with sensor networks. Communications of the ACM Special Issue on Sensor Networks., 47(6), Jun. 2004.

[19] WINS project. Electrical engineering department, UCLA. http://www.janet.ucla.edu/WINS/.

[20] T. Tung and A.Jamalipour. A novel sectional paging strategy for location tracking in cellular networks. Communications Letters, IEEE, 8(1), Jan 2004.

[21] E. W. Weisstein. CRC Concise Encyclopedia of Mathematics. CRC Press, 1969.

[22] B. Xu and O. Wolfson. Time-series prediction with applications to traffic and moving objects databases. In Proc. of ACM international workshop on Data engineering for wireless and mobile access, pages 56-60, 2003.

[23] Y. Xu and W.-C. Lee. On localized prediction for power efficient object tracking in sensor networks. In Proc. 1st International Workshop on Mobile Distributed Computing, pages 434-439, Providence RI, May 2003.

[24] Y. Xu, J. Winter, and W.-C. Lee. Prediction-based strategies for energy saving in object tracking sensor networks. In Proc. International Conference on Mobile Data Management, pages 346-357, Berkeley, CA, Jan. 2004.

[25] Y. Yao and J. Gehrke. Query processing for sensor networks. In Proceedings of the First Biennial Conference on Innovative Data Systems Research, pages 21-32, Asilomar, CA, 2003.

[26] L. C. Zhong, R. Shah, C. Guo, and J. Rabaey. An ultralow power and distributed access protocol for broadband wireless sensor networks. In IEEE Broadband Wireless Summit, Las Vegas, N.V., May 2001.

[27] Y. Zhu and F. Mueller. Feedback edf scheduling exploiting dynamic voltage scaling. In Proc. IEEE Real-Time and Embedded Technology and Applications Symposium, Toronto, Canada, May 2004. 